\title{
STABILITY OF MOBILE MANIPULATORS
}

M.C. Wanner

K.D. Rupp

FhG - IPA stuttgart

Nobelstraße 12

7000 stuttgart 80

Germany

ABSTRACT
Mobile manipulators with very large reaches and high payloads like telescopic cranes or concrete booms are important machines for the construction industry. Those systems have to be very safe. one important item is to ensure stability under any condition.

This paper describes an approach to control the stability considering ground conditions. The mathematical solution as well as the sensor requirements is described.

\section{INTRODUCTION}

Currently most of the mobile construction machines equipped with outriggers do not have any control to ensure their stability - the operator has to decide by experience if the current machine condition ensures the required stability.

An approach to overcome parts of the problem is outlined in $|1|$. With such a system the computer controls if the center of gravity remains within the outrigger feets while the manipulator is moving. If the center of gravity moves into a dangerous area a warning is given to the operator.

For static conditions such an approach is acceptable, however dynamic effects (variations in the ground conditions or within the outriggers) are not detected as they do not create deviations of the center of gravity. A method to overcome parts of the problems is described.

\section{DESCRIPTION OF THE METHOD}

\subsection{Description of the model}

With our method we do first observe the center of gravity and second use the force and torque equations for modelling the system behaviour. To describe the physical systems we use the model as outlined in Fig. 1.

The coordinates of the center of gravity can not be measured, but it is possible to set up the force and torque equations by the input of static pressure in the outrigger feets of the manipulator. We get simple equations for the forces $F_{i}$ and the pressures $P_{i}$ in the outrigger feets:

$\mathrm{F}_{\mathrm{i}}=\mathrm{k}_{\mathrm{i}} \mathrm{P}_{\mathrm{i}}$ 


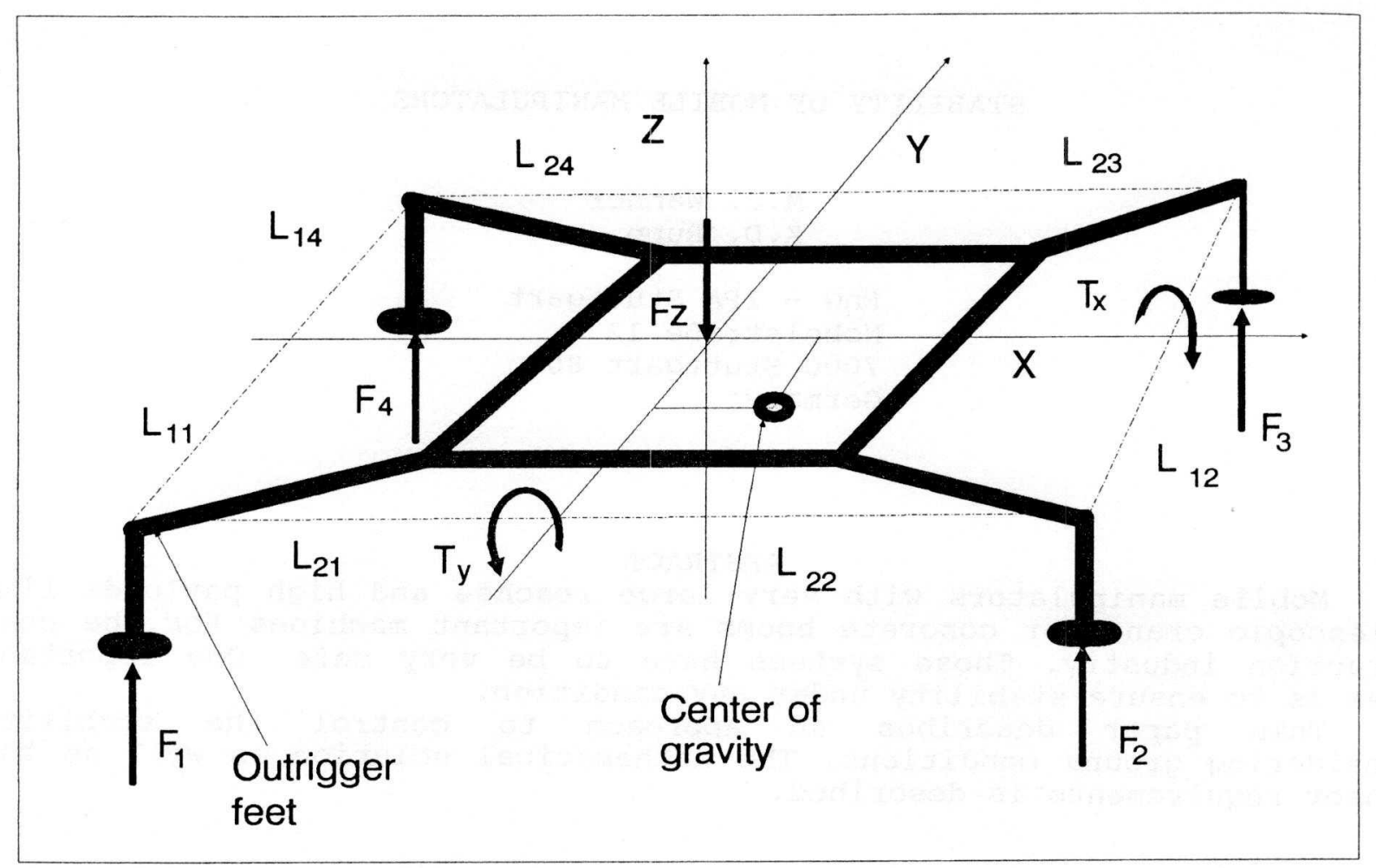

Fig. 1 Geometric model of the manipulator

Using equation (1) the force and torque equation gets to

$$
\begin{aligned}
& {\left[\begin{array}{c}
\mathrm{T}_{\mathrm{X}} \\
\mathrm{T}_{\mathrm{Y}} \\
\mathrm{F}_{\mathrm{z}}
\end{array}\right]=\left[\begin{array}{cccc}
\mathrm{L}_{11} & \mathrm{~L}_{12} & \mathrm{~L}_{13} & \mathrm{~L}_{14} \\
\mathrm{~L}_{21} & \mathrm{~L}_{22} & \mathrm{~L}_{23} & \mathrm{~L}_{24} \\
1 & 1 & 1 & 1
\end{array}\right]\left[\begin{array}{c}
\mathrm{F}_{1} \\
\mathrm{~F}_{2} \\
\mathrm{~F}_{3} \\
\mathrm{~F}_{4}
\end{array}\right]} \\
& \mathrm{T}=\underline{\underline{\mathrm{A}}} \quad \mathrm{F} \\
& \text { with } F_{z}=m g \text { Force caused by weight of the manipulator. } \\
& \text { and TX, Ty Torques caused by the coordinates of center of gra- }
\end{aligned}
$$

Figure 2 shows the block diagram of the stability controller. 


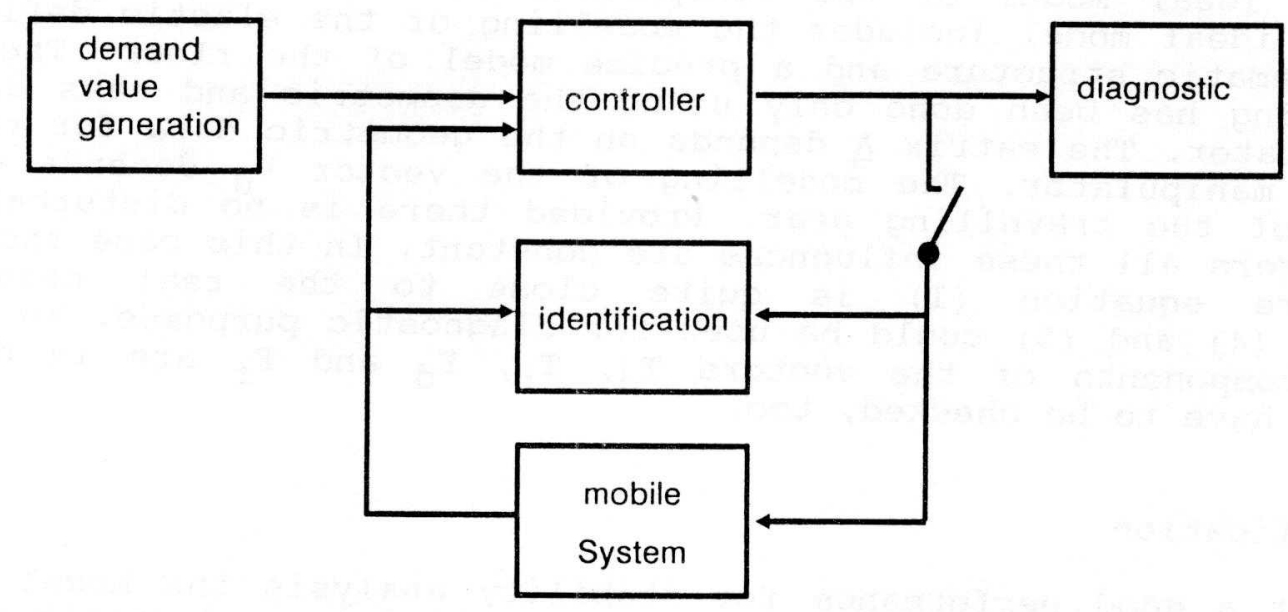

Fig. 2 Block diagram of stability controller

\subsection{Optimum force distribution}

For defining the stability a merit function has to be defined. The aim of this step is to balance the forces in the outrigger feets. This constraint means that the highest force we get is the minimum possible force to solve the equation (1). To get a well balanced force vector we need a least square solution for the force and torque equation (2). A simple way to get a solution is to use the pseudoinverse matrix $\underline{\underline{A}}^{+}$to the matrix $\underline{\underline{A}}$. With a geometric model of the manipulator describing the matrix $\stackrel{A}{=}$ it is possible to compute the vector $T$ and with matrix $\underline{\underline{A}}^{+}$a least square solution for $\mathrm{F}_{\mathrm{d}}$ can be computed.

$\mathrm{F}_{\mathrm{d}}=\underline{\underline{\mathrm{A}}}^{+} \mathrm{T}$

$F_{d}$ is a demand force vector to a stability controller. Therefore the demand value generation includes the merit function for the stability of mobile systems.

\subsection{Model error} we have

If the manipulator is set up with a well balanced force vector $F_{r}$

$$
\left\|F_{d}-F_{r}\right\|<\epsilon_{1}
$$

By moving the axes of the manipulator the vector $F_{d}$ as well as the vector $F_{d}$ changes. But the unequation (4) is always satisfied. The vector $\mathrm{T}_{\mathrm{r}}$ can be computed by a geometric model of the manipulator using equation (2) with the measured forces $F_{r} \cdot T_{d}$ can be computed as a function of geometric and mass data of the manipulator. We get the equations

$$
\begin{aligned}
& \mathrm{T}_{\mathrm{r}}=\underline{\underline{A}} \mathrm{~F}_{\mathrm{r}} \\
& \mathrm{T}_{\mathrm{d}}=\mathrm{f}(\mathrm{m}, \mathrm{g}) \\
& \left\|\mathrm{F}_{\mathrm{d}}-\mathrm{F}_{\mathrm{r}}\right\|<\epsilon_{2}
\end{aligned}
$$


If an ideal model of the manipulator is used, then $\epsilon_{1}=0$ and $\epsilon_{2}=0$. An ideal model includes the modelling of the elastic deflection in the kinematic structure and a precise model of the floor. Therefore the modelling has been done only using the geometric and mass data of the manipulator. The matrix $A$ depends on the geometric data for the set up of the manipulator. The modelling of the vector $F_{d}$ depends on the influence of the travelling gear. Provided there is no disturbance in the outriggers all these influences are constant. In this case the force to pressure equation (1) is quite close to the real behaviour. Unequation (4) and (5) could be used for diagnostic purposes. To ensure that the components of the vectors $T_{d}, T_{r}, F_{d}$ and $F_{r}$ are in defined area. They have to be checked, too.

\subsection{Identification}

To get a good performance for stability analysis the model errors must be eliminated. This can be done for static and quasi-static errors. Using equation (5) and (6) the equation

$\left(\mathrm{T}_{\mathrm{r}}-\mathrm{T}_{\mathrm{d}}\right)=\mathrm{T}_{\mathrm{e}}$

can be set up. To eliminate dynamic errors we use the equation

$\frac{\delta T_{e}}{\delta t}=T_{e}+k\left(T_{d}-T_{r}\right)$

With equation (9) a quasi-static offset $\mathrm{T}_{e}$ could be used to get a better formulation for equation (7):

$\left\|\mathrm{T}_{\mathrm{r}}-\left(\mathrm{T}_{\mathrm{d}}+\mathrm{T}_{\mathrm{e}}\right)\right\|<\epsilon_{2}$

The coefficients of the matrix $A$ could be identified using the vectors $T_{d}(t)$ and $F_{r}(t)$ over some sampling steps.

$$
\begin{aligned}
& {\left[\begin{array}{c}
\mathrm{T}_{\mathrm{d}}(i) \\
\mathrm{T}_{\mathrm{d}}(i+1) \\
\dot{\cdot} \\
\mathrm{T}_{\mathrm{d}}(i+n)
\end{array}\right]=\left[\begin{array}{c}
\underline{\underline{A}} \\
\underline{\underline{A}} \\
\dot{\dot{A}}
\end{array}\right]\left[\begin{array}{c}
\mathrm{F}_{\mathrm{r}}(\mathrm{i}) \\
\mathrm{F}_{\mathrm{r}}(i+1) \\
\dot{\underline{\underline{A}}} \\
\mathrm{~F}_{\mathrm{r}}(\mathrm{n})
\end{array}\right]} \\
& \mathrm{T}^{\wedge} \quad=\quad \underline{\underline{A}}^{\wedge} \quad \mathrm{F}^{\wedge}
\end{aligned}
$$

\subsection{Requirements in hardware}

For the realization of the described algorithms we require a pressure transducer in each leg ( 4 units). It must be ensured that the pressure in each leg is equivalent to the force. In addition two inclinometers to measure the two angels in reference to the horizontal level are required.

Redundant data may be obtained while using the position signals of the manipulator to calculate the center of gravity. Such a scenario is available with the Putzmeister FH26 $|2|$. 


\section{SIMULATION RESULTS AND PROBLEMS}

To test the stability controller we have built up a simulation system for the manipulator FH26. In the simulation model the elastostatic model of the manipulator and an elasto dynamic model for the floor were set up. The stability controller were added to the simulation system and some disturbances were set to the simulation system. These disturbances were a change in floor parameter and chances in parameters and offsets to parameters dealing with the manipulator ontriggers. Figure 3 shows the influence of a floor lowering to the norm of the vector $\mathrm{T}_{\mathrm{e}}$.

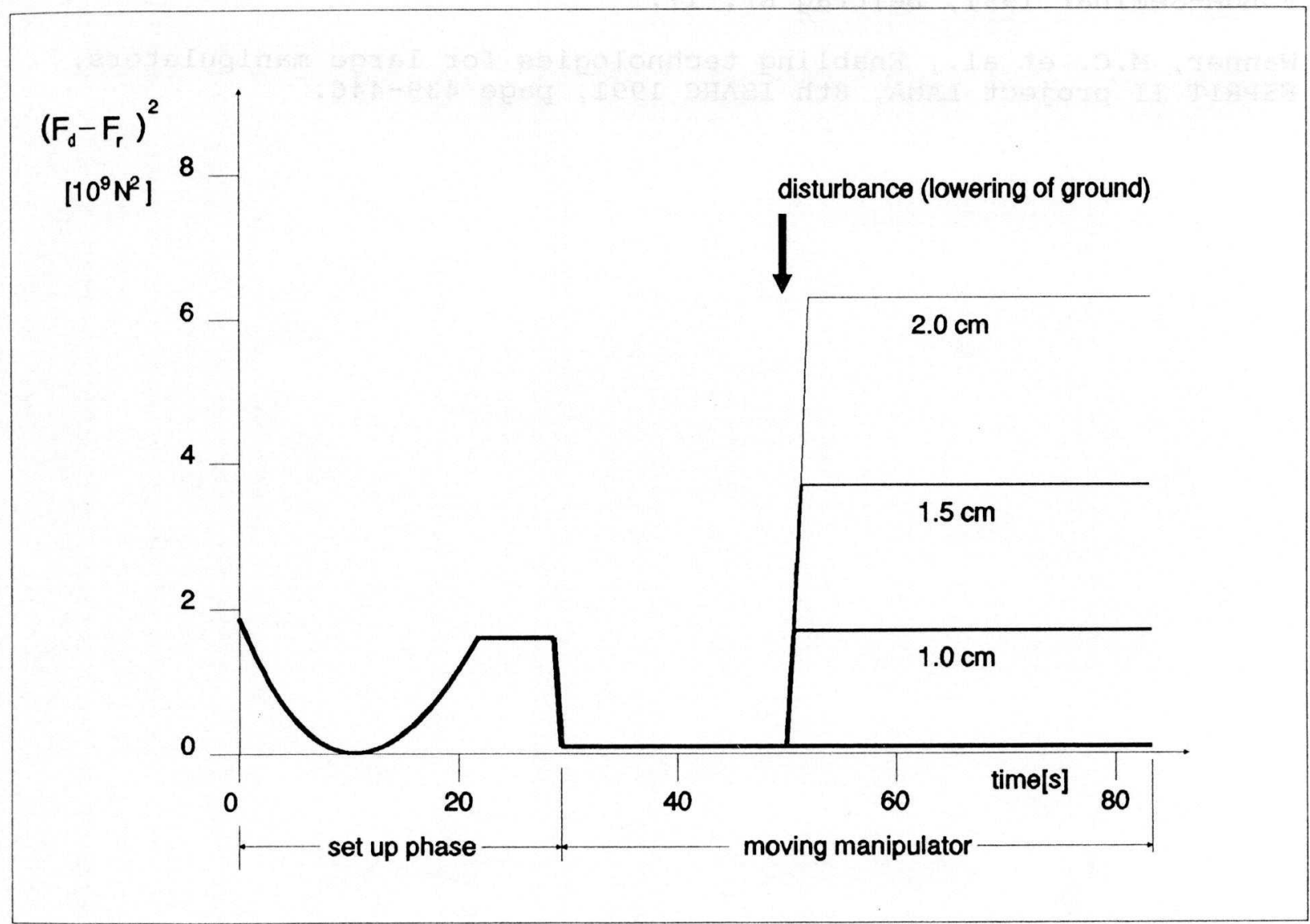

Fig. 3 Simulation of manipulator movement

The stability controller works not exact if any component of the force vector $F_{d}$ is less than zero. In that case one redundant degree of freedom disappears. The identification of tha matrix $\underline{\underline{A}}$ and the vector $\mathrm{T}_{\mathrm{e}}$ is not possible any longer. We have to choose a sub-optimum of the vector $F_{d}$ and to control the vector $F_{r}$ to fit your merit function. During the phase of active controlling the outriggers without modelling the elastic deflections of the outriggers the supervision gets errors. Working in the suboptimum of the vector $F_{d}$ the identification of the quasi-static model errors gets more sensible. 
4. OUTLOOK

For the future we have to perform more practical tests with the Mock Up FH26 to validate the described approach in more detail. The results obtained so far look promising to continue the described way.

\section{References}

|1| Morath, E., Die Litronic im Fahrzeugkran VDBUM-Seminar 1991, Beitrag Nr. 17.

|2| Wanner, M.C. et al., Enabling technologies for large manipulators, ESPRIT II project LAMA, 8th ISARC 1991, page 439-446. 